\title{
Sexually Dimorphic Effects of Melatonin on Brain Arginine Vasotocin Immunoreactivity in Green Treefrogs (Hyla cinerea)
}

\author{
Deborah I. Lutterschmidt Walter Wilczynski \\ Neuroscience Institute, Georgia State University, Atlanta, Ga., USA
}

\section{Key Words}

Melatonin · Arginine vasotocin - Immunoreactivity

Testosterone $\cdot$ Estradiol $\cdot$ Sex differences $\cdot$ Amphibian

\begin{abstract}
Arginine vasotocin (AVT) and its mammalian homologue, arginine vasopressin (AVP), regulate a variety of social and reproductive behaviors, often with complex species-, sex- and context-dependent effects. Despite extensive evidence documenting seasonal variation in brain AVT/AVP, relatively few studies have investigated the environmental and/or hormonal factors mediating these seasonal changes. In the present study, we investigated whether the pineal hormone melatonin alters brain AVT immunoreactivity in green treefrogs (Hyla cinerea). Reproductively active male and female frogs were collected during the summer breeding season and a melatonin-filled or blank silastic capsule was surgically implanted subcutaneously. The duration of hormone treatment was 4 weeks, at which time frogs were euthanized and the brains and blood collected and processed for AVT immunohistochemistry and steroid hormone assay. We quantified AVT-immunoreactive (AVT-ir) cell bodies in the nucleus accumbens (NAcc), caudal striatum and amygdala (AMG), anterior preoptic area, suprachiasmatic nucleus (SCN) and infundibular region of the ventral hypothalamus. Sex differences in AVT-ir cell number were observed in all
\end{abstract}

brain regions except in the anterior preoptic area and ventral hypothalamus, with males having more AVT-ir cells than females in the NAcc, amygdala and SCN. Brain AVT was sensitive to melatonin signaling during the breeding season, and the effects of melatonin varied significantly with both region and sex. Treatment with melatonin decreased AVT immunoreactivity in both the NAcc and SCN in male $H$. cinerea. In contrast, brain AVT was relatively insensitive to melatonin signaling in females, indicating that the regulation of the AVT/AVP neuropeptide system by melatonin may be sexually dimorphic. Finally, melatonin did not significantly influence testosterone or estradiol concentrations of male or female frogs, respectively, suggesting that the effects of melatonin on AVT immunoreactivity are independent of changes in gonadal sex steroid hormones. Collectively, our results indicate that the AVT/AVP neuronal system may be an important target for melatonin in facilitating seasonal changes in reproductive physiology and social behavior.

Copyright $\odot 2012$ S. Karger AG, Basel

\section{Introduction}

Arginine vasotocin (AVT) and its mammalian homologue, arginine vasopressin (AVP), are peptides that function both as neuromodulators and as peripherally acting hormones. The AVT/AVP system regulates a vari-

\section{KARGER \\ Fax +4161306 1234 \\ E-Mail karger@karger.ch}

www.karger.com
(C) 2012 S. Karger AG, Basel

0006-8977/12/0803-0222\$38.00/0

Accessible online at:

www.karger.com/bbe
Deborah I. Lutterschmidt

Department of Biology, Portland State University

PO Box 751

Portland, OR 97207 (USA)

Tel. +1 503725 2325, E-Mail d.lutterschmidt@pdx.edu 
ety of social and reproductive behaviors, often with complex species-, sex- and context-dependent effects [reviewed in Goodson and Bass, 2001]. In anuran amphibians, AVT regulates the production of advertisement calls, the social signals used by male frogs to attract females and make their presence known to conspecific males. Treatment with exogenous AVT stimulates male calling behavior in a number of anurans, including green treefrogs (Hyla cinerea) [Penna et al., 1992; Burmeister et al., 2001], bullfrogs (Rana catesbeiana) [Boyd, 1994], Great Plains toads (Bufo cognatus) [Propper and Dixon, 1997], and gray treefrogs (Hyla versicolor) [Semsar et al., 1998; Tito et al., 1999; Klomberg and Marler, 2000]. In cricket frogs (Acris crepitans), AVT increases the dominant frequency (defined as the spectral frequency with the most energy) of male calls and induces the production of advertisement calls characteristic of less aggressive males [Marler et al., 1995; Chu et al., 1998]. Similarly, AVT increases the call rate in túngara frogs (Physalaemus pustulosus) and causes males to add additional components (i.e. chucks) to their calls, resulting in calls that are potentially more attractive to females [Kime et al., 2007; but see also Kime et al., 2010].

Differences in the behavioral sensitivity to AVT/AVP both between sexes and across social contexts have prompted numerous investigations into whether such differences reflect natural variation in the distribution and abundance of AVT/AVP neurons in the brain. In addition, because reproductive behavior is regulated seasonally in most vertebrates, many studies have examined whether seasonal changes in AVT/AVP contribute to the seasonal expression of reproductive behavior. Collectively, seasonal and sex differences in the AVT/AVP neuropeptide system have been documented in a large number of vertebrate groups, although the exact patterns of these differences vary with species-specific social, reproductive and ecological parameters [Goodson and Bass, 2001; Wilczynski et al., 2005; Adkins-Regan, 2009; Godwin, 2010]. For example, sexually dimorphic patterns of AVT/ AVP neurons in a number of brain areas are almost always in the direction of males having larger and/or more cells than females [Goodson and Bass, 2001]. However, in socially polygamous halfspotted gobies (Asterropteryx semipunctata), females have a greater number of and larger AVT-immunoreactive (AVT-ir) cells in the preoptic area (POA) than male fish [Maruska et al., 2007]. Seasonal changes in AVT immunoreactivity were also observed in halfspotted gobies, with both female and male fish having more and/or larger AVT cells during nonspawning versus spawning periods [Maruska et al., 2007].

Melatonin Alters Brain AVT Cell Number
Similarly, male green treefrogs (H. cinerea) showed an increase in the number of AVT-ir cells at the end of the breeding season in the nucleus accumbens (NAcc), an area implicated in regulating social behavior in vertebrates [O’Bryant and Wilczynski, 2010].

Despite extensive evidence documenting seasonal variation in brain AVT/AVP, relatively few studies have investigated the environmental and/or hormonal factors mediating these seasonal changes. Numerous studies have implicated sex steroid hormones in regulating concentrations of both AVT/AVP as well as AVT/AVP receptors [Goodson and Bass, 2001; De Vries and Panzica, 2006]. However, gonadal hormones are themselves subject to seasonal regulation. In at least one amphibian species, seasonal changes in AVT-ir cell number and size are not influenced by social stimulation or socially induced increases in androgen concentrations [O'Bryant and Wilczynski, 2010]. Thus, the environmental and hormonal factors that regulate seasonal changes in AVT/AVP concentrations remain unclear.

In the present study, we investigated the influence of the pineal hormone melatonin on AVT-ir cell number in green treefrogs. Melatonin is a likely candidate for regulating seasonal changes in AVT/AVP because its production is directly sensitive to environmental cues such as photoperiod and temperature [Axelrod, 1974; Cassone and Natesan, 1997; Prendergast, 2005]. Thus, melatonin synthesis varies seasonally, with overall higher plasma concentrations occurring in response to the shorter photoperiods typical of winter months. Melatonin also plays a role in regulating reproduction in many animals [Tamarkin et al., 1985; Mayer, 1997; Revel et al., 2009]. For example, melatonin influences courtship behavior, gonadal physiology and sex steroid hormone concentrations in a number of ectothermic vertebrates [Mayer, 1997; Krohmer and Lutterschmidt, 2011]. However, studies investigating the influence of melatonin on the AVT/AVP neuropeptide system, particularly in the context of seasonal reproductive behavior, are lacking. Given that (1) AVT/AVP plays an important role in regulating sociosexual behavior and (2) AVT/AVP concentrations change seasonally, we asked whether melatonin alters the number and/or distribution of AVT-ir neurons in the brain of a seasonally breeding amphibian, which would be a potential mechanism for influencing seasonal reproductive behavior.

Green treefogs $(H$. cinerea) are found throughout the southeastern United States and have a breeding period lasting 2-4 months from approximately April through August, depending on environmental conditions and 
geographic locale. Like many other anurans, courtship behavior in green treefrogs is highly social, with males gathering in lek-type aggregations and producing advertisement calls to attract females. Female green treefrogs do not call, but will approach and contact calling males during mate selection. The reproductive behavior of males is androgen dependent [Burmeister and Wilczynski, 2001], and seasonal changes in androgen concentrations parallel seasonal changes in spontaneous calling behavior [O'Bryant and Wilczynski, 2010]. However, androgen replacement following castration does not consistently restore calling behavior to levels observed in intact males [Burmeister and Wilczynski, 2001], therefore suggesting that androgens are necessary but insufficient for male courtship behavior. These results suggest that other factors must be involved in regulating seasonal changes in social reproductive behaviors. We asked whether melatonin modulates the distribution and/or abundance of AVT neurons in male and female treefrogs as well as sex steroid hormone concentrations during the reproductive season.

\section{Materials and Methods}

These experiments were conducted with adult male and female green treefrogs from July to August 2009. Reproductively active male and female frogs were collected during the summer breeding season from natural field sites located within the Charlie Elliott Wildlife Center near Mansfield, Ga., USA. At Georgia State University, animals were housed in groups of 4-6 conspecific, same-sex frogs in 10-gallon aquaria containing a water dish, hide box and artificial foliage. Water was provided ad libitum and animals were fed vitamin-fortified crickets twice per week. All experimental protocols were approved by the Georgia State University Animal Care and Use Committee and were in compliance with guidelines established by the National Institutes of Health Guide for the Care and Use of Laboratory Animals. Field collection of animals was performed under the authority of the Georgia Department of Natural Resources Scientific Collecting Permit 29-WBH-10-58.

\section{Experimental Design}

Following arrival in the laboratory, all frogs were allowed to acclimatize for approximately 1 week prior to hormone manipulation. Reproductively active frogs ( 16 males and 16 females) were maintained in the laboratory at $24^{\circ} \mathrm{C}$ and at a 14 -hour light/10hour dark photoperiod, consistent with the typical environmental conditions for the time of year (July to August). Within each sex, frogs were randomly assigned to either a melatonin or control treatment ( 8 males and 8 females in each group). The duration of hormone exposure was 4 weeks, and therefore frogs remained in the lab no longer than 5 weeks before tissue sampling.

\section{Hormone Manipulation}

To investigate the influence of elevated melatonin concentrations on AVT immunoreactivity, we used melatonin-filled silastic capsule implants similar to Mendonça et al. [1996] and Lutterschmidt [2006]. Small pieces of silastic tubing (0.76 $\mathrm{mm}$ inner diameter $\times 1.65 \mathrm{~mm}$ outer diameter $\times 5 \mathrm{~mm}$ length) were packed with crystalline melatonin (purchased from Sigma, St. Louis, Mo., USA). Control implants were identical in size to the melatonin implants but remained empty. Both ends of each silastic capsule were sealed with Silastic Brand ${ }^{\circledR}$ medical adhesive (Dow Corning, Midland, Mich., USA).

Frogs were anesthetized by immersion in $0.1 \%$ methylsulfonic acid (MS-222, pH 7.4) for 5-10 min. A small incision (about $2 \mathrm{~mm}$ ) was made on the lateral side of the animal, approximately $1 \mathrm{~cm}$ posterior to the forearm and $20-30 \mathrm{~mm}$ dorsal to the lateral stripe. The white or yellowish lateral stipe is a pattern characteristic of most green treefrogs; it extends from the upper lip posteriorly to the hind limbs on both sides of the body, although the length and presence of the lateral stripe is variable among individuals. A silastic capsule was inserted subcutaneously and the incision was closed using suture and a small amount of tissue glue (Vetbond, Inc.). Frogs were allowed to fully recover before being placed back into their home cages, and all frogs within a cage received the same hormone treatment. Frogs were monitored daily during the 4 -week treatment period to ensure proper healing and that the hormone implants remained intact.

\section{Tissue Processing}

At the conclusion of the 4-week treatment period, frogs were euthanized by immersion in $0.2 \% \mathrm{MS}-222$ ( $\mathrm{pH}$ 7.4) for $5 \mathrm{~min}$ and rapidly decapitated. Trunk blood was collected from each frog for hormone assay; samples were stored on ice until centrifuged and the plasma separated. Plasma samples were then stored at $-20^{\circ} \mathrm{C}$ until assayed for hormone concentrations. Brains were immersion fixed in $4 \%$ paraformaldehyde in $0.1 \mathrm{M}$ phosphate buffer for $20 \mathrm{~h}$ at $4^{\circ} \mathrm{C}$, cyroprotected in $30 \%$ sucrose in $0.1 \mathrm{M}$ phosphate buffer, and sectioned on a cryostat (Leica 3050S). Brains were sectioned into three series of $25-\mu \mathrm{m}$ coronal sections that were thaw-mounted onto subbed slides (Fisherbrand: Superfrost Plus). Slides were stored at $-20^{\circ} \mathrm{C}$ until immunohistochemical processing. Gonads were inspected to confirm the sex of each animal.

\section{AVT Immunohistochemistry}

The influence of melatonin on the distribution and abundance of AVT was examined using immunohistochemistry on one series of tissue. All slides were processed simultaneously in one immunohistochemistry assay. Prior to incubation with the primary antibody, slides were first washed 5 times for 5 min each with $0.1 \mathrm{M}$ phosphate-buffered saline (PBS; pH 7.4) followed by quenching in $1 \%$ hydrogen peroxide in PBS for $30 \mathrm{~min}$. Slides were washed in PBS $(5 \times 5 \mathrm{~min})$ and then incubated for $60 \mathrm{~min}$ in PBS containing $10 \%$ normal goat serum, $10 \%$ avidin (Vector Labs, Inc.) and $0.3 \%$ Triton $\mathrm{X}$ to reduce non-specific binding. AVT immunoreactivity was examined using a rabbit anti-AVT antiserum (generously provided by Dr. Matthew S. Grober, Georgia State University) at a dilution of 1:5,000 in PBS containing $10 \%$ normal goat serum, $10 \%$ biotin (Vector Labs, Inc.) and $0.3 \%$ Triton X. Sections were incubated with primary antibody for $48 \mathrm{~h}$ at $4{ }^{\circ} \mathrm{C}$ in a humid chamber. The slides were then washed in PBS $(5 \times 5 \mathrm{~min})$ and the primary antibody signal amplified by incubation for $60 \mathrm{~min}$ with 
biotinylated goat anti-rabbit secondary antibody (Vector Labs, Inc.) diluted $1: 400$ in $0.3 \%$ Triton $\mathrm{X}$ in PBS. Following incubation with the secondary antibody, the sections were again washed in PBS $(5 \times 5 \mathrm{~min}$ ), incubated for $60 \mathrm{~min}$ in avidin conjugated to horseradish peroxidase (Elite ABC peroxidase kit, Vector Laboratories, Inc.), and rinsed in PBS ( $\times 5 \mathrm{~min})$. Primary antibody binding was visualized using $0.25 \mathrm{mg} / \mathrm{ml}$ diaminobenzidine in $0.2 \%$ hydrogen peroxide in $0.1 \mathrm{M}$ Tris- $\mathrm{HCl}$ buffer. The reaction was terminated by immersion in nanopure $\mathrm{H}_{2} \mathrm{O}(3 \times 5 \mathrm{~min})$ followed by dehydration in a graded ethanol series. The tissue was cleared with Citrisolv (Fisher Scientific, Pittsburg, Pa., USA) and the slides were covered with permount and coverslips.

\section{Immunoreactive Cell Counting}

Stained tissue was examined with an Olympus BX41 microscope using a Slide Pursuit digital camera (Spot Pursuit, Diagnostic Instruments) and Spot Basic imaging software (Diagnostic Instruments). Locations of AVT-ir cells were mapped onto standard anatomical frog brain sections adapted from Northcutt and Kicliter [1980] and Neary and Northcutt [1983]. Immunoreactive cells were counted manually; animals were coded so that the observer was blind to the treatment group and sex of individuals. If a tissue section was missing or damaged, the section was assigned the mean cell count of the preceding and succeeding sections. If two consecutive sections were missing and/or damaged, the animal was eliminated from subsequent analyses. Final sample sizes for each category of treatment and sex within each region of interest are given in the figures.

For all regions except the anterior preoptic area (POA), quantification of immunoreactive cells in each tissue section was performed first under $200 \times$ magnification and again under $400 \times$ magnification. These counts were usually identical, but in some cases, they differed by 1-3 cells. In these instances, the section was recounted under $400 \times$ magnification to verify the cell count. Due to the much higher density of AVT-ir cells within the POA (i.e. $>600$ cells per individual), cells in this region were quantified twice under $400 \times$ magnification and each section was then assigned the mean cell count. Replicate cell counts never differed by more than $5 \%$.

The number of AVT-ir cells in each region was quantified in one brain series and totaled for each individual. Because tissue was divided into 3 different series of $25-\mu \mathrm{m}$ sections, approximately $75 \mu \mathrm{m}$ separated each section within a series. In the regions of interest, anuran AVT-ir cells are approximately $10 \mu \mathrm{m}$ in diameter. Thus, there is little possibility that double counting of cells split between two sections inflated our cell quantifications. In addition, there is no reason to suspect that double counting would occur more frequently in any of the treatment categories. Finally, a DAB-labeled cell was counted only if the cell was of the same general size and shape as the other AVT-ir cells in the region of interest, thereby excluding partial cells from our quantifications.

\section{Hormone Assays}

Plasma concentrations of testosterone in male frogs and estradiol in female frogs were analyzed using commercial enzyme immunoassay kits (Cayman Chemical, Ann Arbor, Mich., USA) following procedures previously validated for green treefrogs [e.g., see Lynch and Wilczynski, 2005; Almli and Wilczynski, 2009]. Briefly, plasma volumes were approximately $16 \mu \mathrm{l}$ for male and 50 $\mu \mathrm{l}$ for female samples. Following plasma dilution (1:50 for testosterone, 1:6 for estradiol), approximately 2,500 cpm of tritiated hormone was added to each sample to determine individual sample recovery. Samples were extracted with $3 \mathrm{ml}$ of anhydrous ethyl ether, and the ether phase was removed and dried under nitrogen gas in a $37^{\circ} \mathrm{C}$ water bath. Extracted and reconstituted samples were then applied to the enzyme immunoassay plates in triplicate according to manufacturer instructions. All samples were corrected for individual recovery variation. Mean extraction efficiency was $97 \%$ for testosterone and $89 \%$ for estradiol. Mean intra- and interassay coefficients of variation were 5.6 and $14.2 \%$ for testosterone and 5.3 and $13.2 \%$ for estradiol, respectively.

\section{Statistics}

We first performed a multifactor multivariate analysis of variance (MANOVA) to determine if melatonin alters the number of AVT-ir cells in green treefrogs. For this analysis, the five regions of interest were treated as multiple response variables, and treatment and sex were included as between-subject factors. Because changes in one AVT-ir cell population may be associated with changes in other AVT-ir regions within the same individual, a MANOVA was used to simultaneously evaluate the effects of melatonin on all AVT-ir cell populations. This analysis was used to identify which cell populations contributed to the significant main effects of the MANOVA. Due to missing sections and/or tissue damage, it was not possible to quantify all five regions of interest in every individual. Thus, the sample sizes available for MANOVA were reduced because the analysis discards an animal if data for any region are missing within that individual. Effective sample sizes for the MANOVA were as follows: female control, 5; female melatonin, 8; male control, 5; male melatonin, 6 . When MANOVA indicated a significant main effect of the sex $\times$ treatment interaction term, we then performed a two-factor ANOVA followed by Tukey's multiple comparison test within each region to further investigate these differences while maximizing sample sizes and statistical power.

To determine if melatonin alters sex steroid hormone concentrations, we used a t test to examine differences in plasma testosterone and estradiol between treatments in male and female frogs, respectively. Where necessary, data were transformed using natural log or square root functions to meet the parametric requirements of normality and equal variance. All statistical comparisons were considered significant at $\mathrm{p} \leq 0.05$.

\section{Results}

We observed five distinct regions of AVT-ir staining in male and female frogs (fig. 1). Similar to O'Bryant and Wilczynski [2010], cell bodies were quantified in the NAcc, central and medial amygdala (AMG), anterior POA, suprachiasmatic nucleus (SCN), and infundibular region of the ventral hypothalamus $(\mathrm{VH})$. Immunoreactive cell bodies were also observed in the caudal striatum, but it was difficult to establish boundaries for this cell population that were distinct from both the central and medial AMG. Thus, AVT-ir cells located in the caudal 
striatum were grouped into and analyzed with the AMG. Similarly, we could not establish consistently distinct boundaries between cells in the anterior POA and the magnocellular region of the POA due to the very high numbers of cells in these areas. Thus, our reported cell counts for the POA represent both the anterior POA and the magnocellular region of the POA. As expected, omission of the primary antibody resulted in an absence of immunoreactive staining.

A two-way MANOVA indicated that the number of AVT-ir cells varied significantly with sex $\left(\mathrm{F}_{5,16}=4.295\right.$, $\mathrm{p}=0.011)$. Although the main effects of treatment were not statistically significant $\left(\mathrm{F}_{5,16}=1.140, \mathrm{p}=0.380\right)$, a significant interaction between sex and treatment was observed $\left(\mathrm{F}_{5,16}=6.390, \mathrm{p}=0.002\right)$. Tests of between-subject effects for each region are provided in the following sections. When a significant main effect of the sex $\times$ treatment interaction term was detected by MANOVA, the data were further analyzed by two-factor ANOVA within each region to maximize sample sizes.

\section{Nucleus Accumbens}

Neither sex $\left(\mathrm{F}_{1,24}=0.799, \mathrm{p}=0.382\right)$ nor treatment $\left(\mathrm{F}_{1,24}=0.732, \mathrm{p}=0.403\right)$ significantly influenced the number of AVT-ir cells in the NAcc. However, we observed a significant interaction between these factors $\left(\mathrm{F}_{1,24}=7.311, \mathrm{p}=0.014\right.$, from a two-factor MANOVA $)$. Within the NAcc, we then used a two-factor ANOVA to further analyze the significant main effect of the sex $\times$ treatment interaction while maximizing sample sizes. Similar to the results of the MANOVA, the main effects of sex $\left(\mathrm{F}_{1,29}=0.463, \mathrm{p}=0.502\right)$ and treatment $\left(\mathrm{F}_{1,29}=\right.$ $0.221, \mathrm{p}=0.642$ ) from a two-way ANOVA were not statistically significant. A significant interaction between sex and treatment was observed $\left(\mathrm{F}_{1,29}=0.023, \mathrm{p}=0.023\right)$, indicating that the effect of melatonin depends on sex. Within the control treatment, Tukey's multiple comparisons test indicated that breeding male frogs have significantly more AVT-ir cells in the NAcc than female frogs (fig. 2). In addition, while melatonin does not influence AVT immunoreactivity in female frogs, it significantly reduced the number of AVT-ir cells in the NAcc of male frogs during the summer breeding season (fig. 2).

\section{Central and Medial AMG and Caudal Striatum}

Results from a two-way MANOVA indicate that the number of AVT-ir cells varied significantly with sex $\left(\mathrm{F}_{1,24}=18.948, \mathrm{p}<0.001\right)$, with male frogs having more AVT-ir cells in the central and medial AMG than female
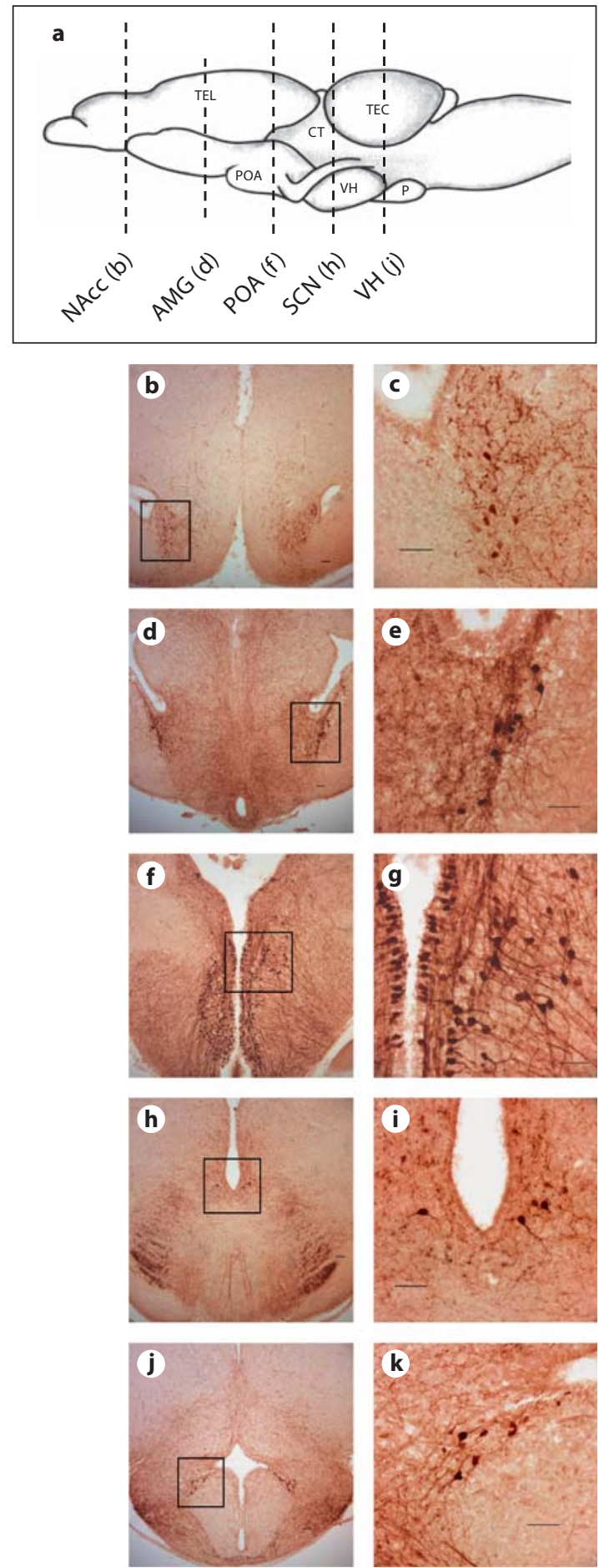

Fig. 1. Distribution of AVT immunoreactivity in green treefrogs (H. cinerea). a Schematic showing the sagittal view of the frog brain; the rostral portion of the brain is oriented to the left. The vertical lines indicate the level of sections through AVT-ir cell populations. Example photomicrographs of brain sections are shown for the NAcc (b, c), AMG (d, e), anterior POA (f, g), SCN $(\mathbf{h}, \mathbf{i})$ and infundibular region of the $\mathrm{VH}(\mathbf{j}, \mathbf{k})$. Scale bars $=50 \mu \mathrm{m}$. Boxes delineate magnified spaces. 


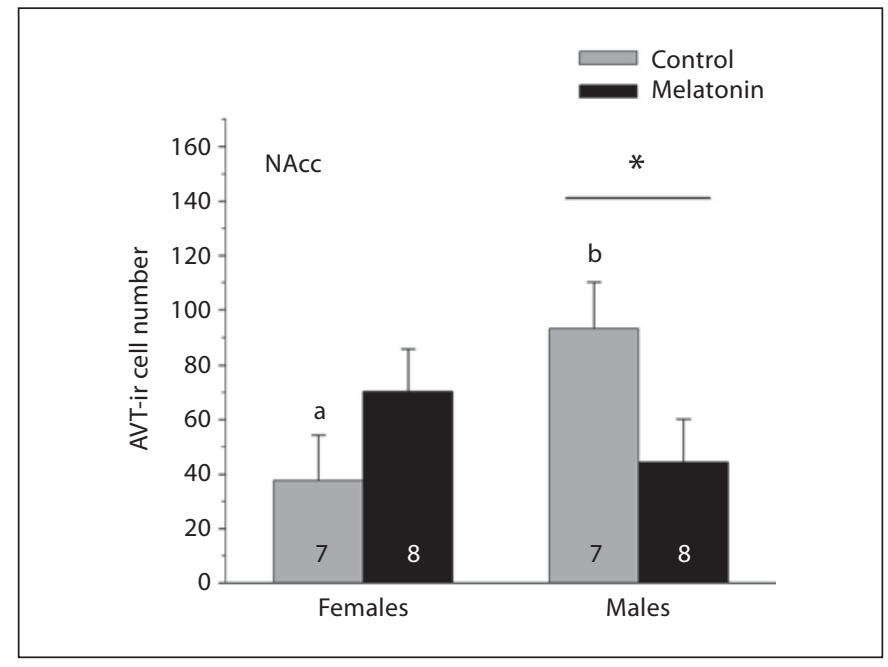

Fig. 2. Influence of melatonin on AVT-ir cell number within the NAcc of male and female green treefrogs during the summer reproductive period. Data are the mean number of AVT-ir cells +1 $\mathrm{SE}$; sample sizes are listed above the $\mathrm{x}$-axis. Letters indicate significant sex differences in AVT-ir cell number within the control treatment. The asterisk above the horizontal line indicates a significant effect of melatonin within males (results from a two-factor ANOVA followed by Tukey's multiple comparison procedure).

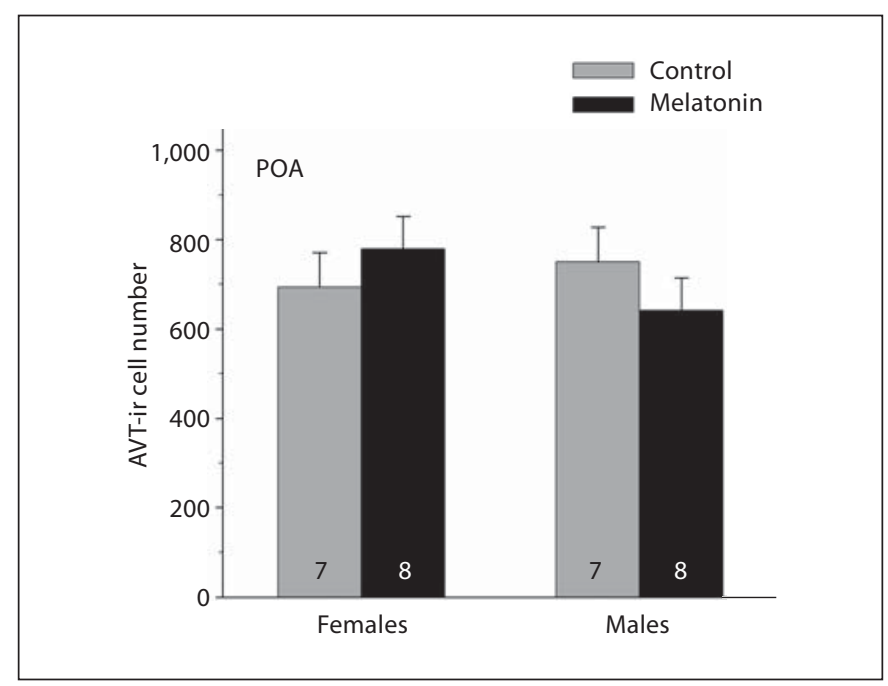

Fig. 4. Influence of melatonin on AVT-ir cell number within the anterior POA and magnocellular region of the POA of male and female green treefrogs during the summer reproductive period. Data are the mean number of AVT-ir cells + 1 SE; sample sizes are listed above the $\mathrm{x}$-axis.

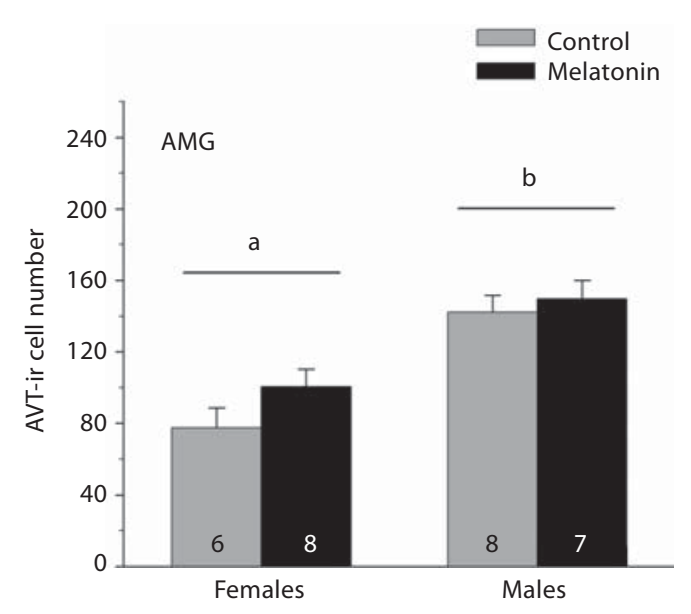

Fig. 3. Influence of melatonin on AVT-ir cell number within the caudal striatum and AMG of male and female green treefrogs during the summer reproductive period. Data are the mean ir-cell number +1 SE; sample sizes are listed above the $\mathrm{x}$-axis. Letters above the horizontal lines indicate a significant sex difference in AVT-ir cell number (results from a two-way MANOVA).

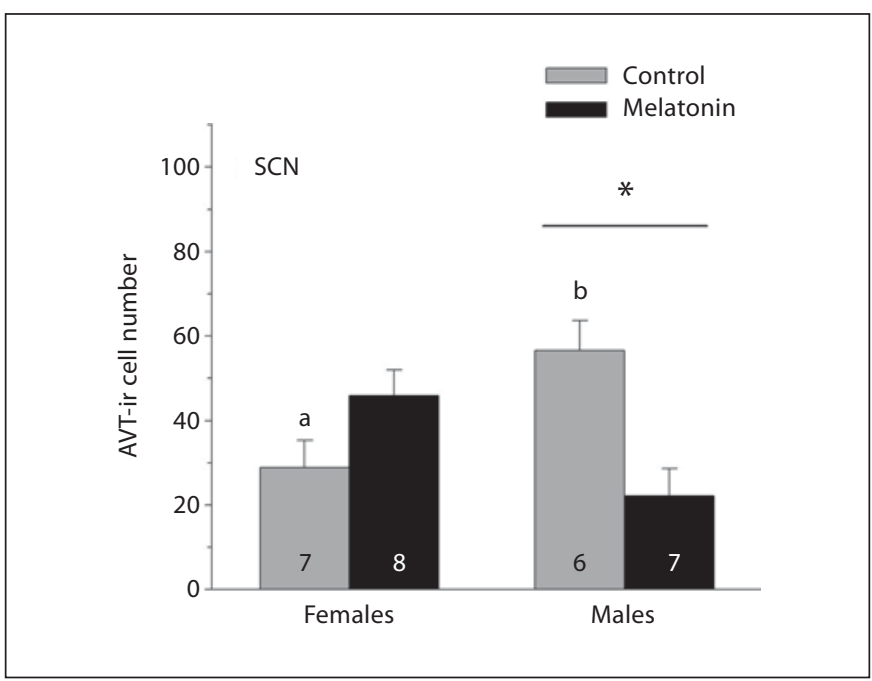

Fig. 5. Influence of melatonin on AVT-ir cell number within the SCN of male and female green treefrogs during the summer reproductive period. Data are the mean number of AVT-ir cells +1 $\mathrm{SE}$; sample sizes are listed above the $\mathrm{x}$-axis. Letters indicate significant sex differences in AVT-ir cell number within the control treatment. The asterisk above the horizontal line indicates a significant effect of melatonin within males (results from a two-factor ANOVA followed by Tukey's multiple comparison procedure). 


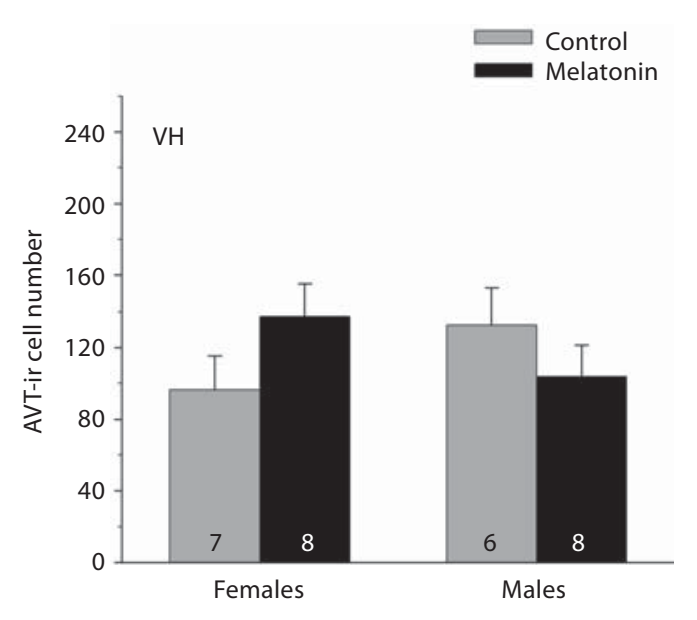

Fig. 6. Influence of melatonin on AVT-ir cell number within the infundibular region of the $\mathrm{VH}$ of male and female green treefrogs during the summer reproductive period. Data are the mean number of AVT-ir cells + 1 SE; sample sizes are listed above the $\mathrm{x}$-axis.

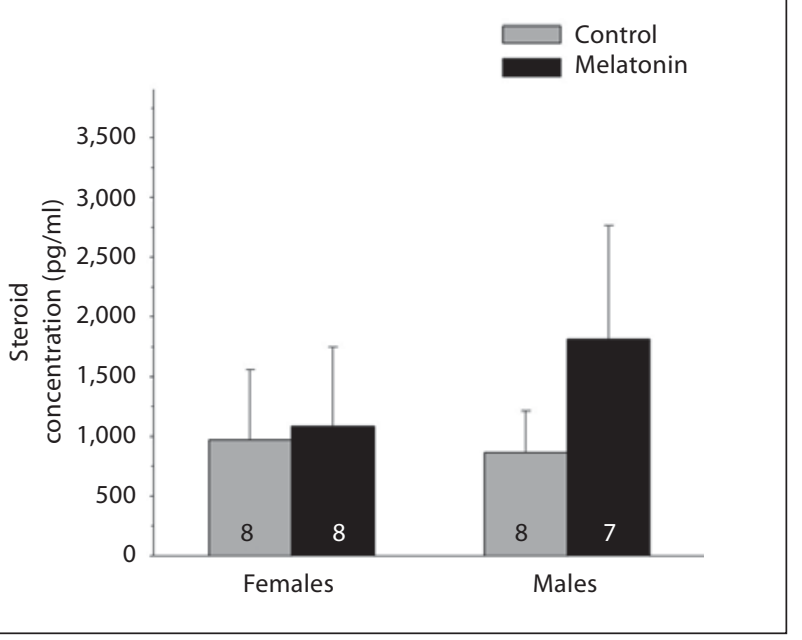

Fig. 7. Influence of melatonin on estradiol in female green treefrogs and testosterone in male frogs during the summer reproductive period. Data are the mean hormone concentration + 1SE; sample sizes are listed above the $\mathrm{x}$-axis. frogs (fig. 3). Melatonin treatment did not significantly influence the number of AVT-ir cells in the AMG $\left(\mathrm{F}_{1,24}=\right.$ $1.151, \mathrm{p}=0.296)$. The interaction between sex and treatment was not statistically significant $\left(\mathrm{F}_{1,24}=0.391, \mathrm{p}=\right.$ 0.539, from a two-way MANOVA).

Anterior POA and Magnocellular Region of the POA

Neither sex $\left(\mathrm{F}_{1,24}=0.010, \mathrm{p}=0.921\right)$ nor melatonin treatment $\left(\mathrm{F}_{1,24}=0.011, \mathrm{p}=0.918\right)$ significantly influenced the number of AVT-ir cells in the anterior POA of breeding frogs (fig. 4). The interaction between sex and treatment was also statistically non-significant $\left(\mathrm{F}_{1,24}=\right.$ $1.856, \mathrm{p}=0.188$, from a two-way MANOVA).

\section{Suprachiasmatic Nucleus}

A two-way MANOVA indicates that neither sex $\left(\mathrm{F}_{1,24}=0.799, \mathrm{p}=0.382\right)$ nor treatment $\left(\mathrm{F}_{1,24}=0.866, \mathrm{p}=\right.$ 0.363 ) significantly influenced the number of AVT-ir cells in the SCN. However, we observed a significant interaction between sex and treatment $\left(\mathrm{F}_{1,24}=16.028, \mathrm{p}=\right.$ 0.001 , from a two-factor MANOVA). Within the SCN, we then used a two-factor ANOVA to further analyze the main effects of the sex $\times$ treatment interaction detected by the multifactor MANOVA. The main effects of treatment $\left(\mathrm{F}_{1,27}=1.795, \mathrm{p}=0.193\right)$ and $\operatorname{sex}\left(\mathrm{F}_{1,27}=0.0951, \mathrm{p}=\right.$ 0.760, from a two-way ANOVA) on AVT-ir cell number in the SCN were not statistically significant, although a significant interaction between treatment and sex occurred $\left(\mathrm{F}_{1,27}=15.707, \mathrm{p}<0.001\right)$. Within the control treatment, a Tukey multiple comparison test indicated that breeding male frogs have significantly more AVT-ir cells in the SCN than female frogs (fig. 5). In addition, while melatonin does not influence AVT immunoreactivity in females, it significantly reduced the number of AVT-ir cells in the SCN of males during the summer breeding season (fig. 5).

\section{Infundibular Region of the $\mathrm{VH}$}

The number of AVT-ir cells in the VH did not vary significantly with sex $\left(\mathrm{F}_{1,24}=0.153, \mathrm{p}=0.700\right)$ or melatonin treatment $\left(\mathrm{F}_{1,24}=0.098, \mathrm{p}=0.757\right.$; fig. 6). The interaction between sex and treatment was also statistically nonsignificant $\left(\mathrm{F}_{1,24}=2.840, \mathrm{p}=0.107\right.$, from a two-way MANOVA).

\section{Sex Steroid Hormones}

Treatment of male and female green treefrogs with melatonin did not significantly alter testosterone $(\mathrm{t}=$ -0.661 ; d.f. $=14 ; \mathrm{p}=0.519)$ or estradiol $(\mathrm{t}=-0.333$; d.f. $=$ $14 ; \mathrm{p}=0.744$ ) concentrations, respectively (fig. 7). 


\section{Discussion}

We demonstrate that the presence and distribution of AVT-ir cells in the brain of green treefrogs (H. cinerea) is sensitive to melatonin signaling during the breeding season. The effects of melatonin varied significantly with both brain region and sex. In summary, sex differences in AVT-ir cell number were observed in all regions except in the anterior POA and $\mathrm{VH}$, with males having more AVT-ir cells than females in the NAcc, AMG and SCN. Treatment with melatonin decreased AVT immunoreactivity in both the neuromodulatory NAcc and the presumed neurosecretory SCN cell populations in male $H$. cinerea. In contrast, brain AVT was relatively insensitive to melatonin signaling in females, indicating that the regulation of the AVT/AVP neuropeptide system by melatonin is sexually dimorphic. Finally, melatonin did not significantly influence testosterone or estradiol concentrations of males or females, respectively, suggesting that the effects of melatonin on AVT immunoreactivity are independent of changes in sex steroid hormones. We suggest that melatonin plays a role in regulating seasonal changes in male social reproductive behavior via changes in AVT signaling.

\section{Sexually Dimorphic Patterns of AVT}

Sex differences in the AVT/AVP neuropeptide system have been documented in a large number of vertebrate groups [Goodson and Bass, 2001; Wilczynski et al., 2005; Adkins-Regan, 2009; Godwin, 2010]. While the nature of these differences tend to vary with social, reproductive and ecological parameters, sexually dimorphic patterns of AVT/AVP neurons are almost always in the direction of males having larger and/or more cells than females [Goodson and Bass, 2001]. In the current study, we demonstrate a similar sexually dimorphic pattern of AVT in the brain of green treefrogs. During the breeding season, male green treefrogs had significantly higher numbers of AVT-ir cells than females in the NAcc, AMG and SCN. These results are similar to those of Marler et al. [1999], who demonstrated that both calling and noncalling satellite male cricket frogs (Acris crepitans) have significantly higher numbers of AVT-ir cells in the NAcc than female frogs. Our study suggests that sexually dimorphic patterns of AVT-ir neurons in $H$. cinerea may be important for regulating sociosexual behavior in males.

Additional studies are needed to understand the hormonal factors that regulate sexually dimorphic patterns of AVT. For example, increased androgen concentrations during the breeding season may regulate changes in the production and/or release of AVT in this and other socially breeding vertebrates. Indeed, increased AVT/AVP levels in the brain are associated with increased androgen concentrations in males across many species [Goodson and Bass, 2001; De Vries and Panzica, 2006; Hillsman et al., 2007; Kabelik et al., 2008]. However, decreased AVP immunoreactivity occurs in male mice lacking a functional gene for aromatase, the enzyme that metabolizes androgens to estrogens [Plumari et al., 2002]. Thus, whether steroid-induced changes in the AVT/AVP neuronal system are related to androgens or their estrogenic metabolites requires further study.

\section{Effects of Melatonin on AVT}

Our data demonstrate that melatonin modulates brain AVT immunoreactivity in green treefrogs, and the effects of melatonin are both region specific and sexually dimorphic. For example, treatment of male frogs with melatonin significantly changed immunoreactive cell number in the NAcc and SCN, but not in the AMG, anterior POA or $\mathrm{VH}$. These results suggest that melatonin receptor distribution varies across these brain regions. In support of this hypothesis, melatonin receptor binding detected by autoradiography in green frogs (Rana esculenta) was limited to the amygdaloid nucleus pars lateralis, striatum and suprachiasmatic area of the hypothalamus [Tavolaro et al., 1995]. However, information regarding the neural distribution of melatonin receptors in other amphibians is limited. Like most ectotherms, anuran amphibians possess three different melatonin receptor subtypes: melatonin $1 \mathrm{a}, 1 \mathrm{~b}$ and $1 \mathrm{c}$ receptors [Wiechmann et al., 2003, 2009; see also Wiechmann et al., 1999; Serino et al., 2011]. No studies have addressed the presence and/or distribution of melatonin receptors in $H$. cinerea. Because melatonin receptor distribution can vary among different species, such studies are needed to understand how melatonin regulates brain AVT in this (and potentially other) species. Additional studies are also needed to confirm that the effects of melatonin on AVT immunoreactivity are indeed receptor mediated.

The present study also demonstrates that the effects of melatonin on AVT-ir cell populations are sexually dimorphic. The AVT neuropeptide system was sensitive to melatonin signaling only in male frogs; melatonin did not significantly influence the number of AVT-ir cells in females during the breeding season. However, it should be noted that in the two areas in which melatonin significantly decreased the number of AVT-ir cells in reproductive males (i.e. the NAcc and SCN), melatonin tended to increase the number of AVT-ir cells in females. Future studies using a 
combination of quantitative approaches (e.g., in situ hybridization, immunohistochemistry and rate of axonal release in cell culture) to address this potential sex difference would help clarify whether melatonin modulates AVT signaling in females. Moreover, additional studies are needed to address how different doses of melatonin, administered at different times of the circadian and circannual cycle, influence the number AVT-ir cells in the brain. For example, it is possible that AVT cells have different sensitivities to melatonin in male and female green treefrogs. Similarly, it is also likely that the sensitivity of AVT neurons to melatonin varies at different times of the annual reproductive cycle. Such studies would be beneficial in understanding how environmental cues, transduced via melatonin cycles, might influence seasonal changes in the AVT/AVP neuropeptide system.

It is interesting to note that melatonin significantly decreased the number of AVT-ir cells in the NAcc of males, a neuromodulatory population of AVT cells associated with male sociosexual behavior in anurans. For example, calling male cricket frogs (A. crepitans) have significantly fewer AVT-ir cells in the NAcc than noncalling satellite males, presumably because calling males exhibit higher AVT release in order to maintain calling behavior [Marler et al., 1999]. In male green treefrogs, only the NAcc population of AVT-ir cells changed significantly toward the end of the breeding season, again suggesting that this AVT cell population is important in regulating seasonal social reproductive behavior in males [O'Bryant and Wilczynski, 2010]. Therefore, our results support the hypothesis that melatonin regulates seasonal changes in male sociosexual behavior via changes in the AVT/AVP brain neuromodulatory system.

Within the SCN, a brain region critical to producing and maintaining daily rhythms in physiology and behavior in mammals, melatonin significantly decreased AVTir staining in male green treefrogs. Additional studies are necessary to understand how melatonin-induced changes in AVT-ir cell number relate to the regulation of biological rhythms by the SCN. For example, AVP neurons within the SCN of mammals are known to be clock controlled, and SCN-derived AVP plays an important role in regulating daily neuroendocrine rhythms important to reproduction and energy balance [reviewed in Kalsbeek, 2010].

Further research is necessary to understand mechanistically why the influence of melatonin on AVT-ir cell number varies with sex and brain region. For example, whether melatonin receptors are colocalized within AVT-ir neurons in these brain regions is unknown. Fur- thermore, it is unknown whether the distribution and/or expression of melatonin receptors in green treefrogs is sexually dimorphic, as has been reported for birds [reviewed in Aste et al., 2001]. While it is possible that male testosterone concentrations regulate the regional distribution of melatonin receptors, available evidence in birds and mammals suggests that melatonin receptor expression is independent of gonadal androgens [Skene et al., 1993; Bentley and Ball, 2000]. Rather, sexually dimorphic melatonin receptor expression in the brain may be regulated by estrogens. In vitro incubation of ovarian tissue of rats with estradiol results in the downregulation of type 1 melatonin receptor [Clemens et al., 2001]. Whether a similar estradiol-mediated mechanism might be involved in establishing sex differences in the distribution of neural melatonin receptors requires further study.

Alternatively, the influence of melatonin on AVT-ir cell number could be androgen mediated. For example, several studies in amphibians indicate that melatonin may regulate seasonal changes in reproduction via decreased gonadosomatic index, spermatogenesis and Leydig cell number and/or activity [reviewed in Mayer et al., 1995]. In green treefrogs, injections of melatonin ( $4 \mu \mathrm{g}$ per day) administered under long-day conditions ( $16 \mathrm{~h}$ light $/ 8 \mathrm{~h}$ darkness) decreased the gonadosomatic index in male and female frogs [de Vlaming et al., 1974]. More recently, O'Bryant and Wilczynski [2010] demonstrated that seasonal changes in AVT-ir cell number in male $H$. cinerea toward the end of the breeding season are concomitant with declining androgen concentrations. Thus, it is possible that melatonin alters brain AVT indirectly by modulating the production of sex steroid hormones. However, our melatonin treatments did not significantly alter either testosterone concentrations in male frogs or estradiol in female frogs. Thus, a direct effect of melatonin on brain AVT, independent of changes in sex steroid hormones, is suggested.

In summary, our results corroborate several lines of evidence suggesting that seasonal changes in the AVT/ AVP system may be regulated by the hormone melatonin [Isobe et al., 2001; Juszczak, 2001; Rasri et al., 2008; Mogulkoc and Baltaci, 2010]. For example, short photoperiod exposure in Djungarian hamsters (Phodopus sungorus), which mimics winter-like environmental conditions, reduces the expression of AVP mRNA in the bed nucleus of the stria terminalis [Rasri et al., 2008]. In rats, melatonin inhibits increased plasma AVP responses to thyroxine treatment in vivo [Mogulkoc and Baltaci, 2010]. In addition, Isobe et al. [2001] demonstrated that melatonin inhibits AVP release from cultured SCN slices 
in rats, and these effects are specifically mediated by the type 2 melatonin receptor. In the present study, it is unknown whether the observed effects of melatonin on AVT-ir staining result from changes in gene expression, peptide production and/or the rate of axonal release. Nevertheless, these studies indicate that melatonin modulates AVT signaling in the brain. How melatonin-induced changes in the AVT/AVP neuropeptide system might in turn regulate seasonal changes in social and reproductive behaviors warrants future attention.

\section{Acknowledgments}

We thank Matthew S. Grober at Georgia State University for the generous donation of rabbit anti-AVT primary antibody. We also thank Michael Black, Javontè Carter, Anna Creighton, Leslie Dunham, and Jemetrius Meyers for their assistance with these studies. This work was funded by the Center for Behavioral Neuroscience and the National Institutes of Health (F32 GM083888 to D.I.L. and R01 MH057066 to W.W.).

\section{References}

Adkins-Regan E (2009): Neuroendocrinology of De Vries GJ, Panzica GC (2006): Sexual differensocial behavior. ILAR J 50:5-14.

-Almli LM, Wilczynski W (2009): Sex-specific modulation of cell proliferation by socially relevant stimuli in the adult green treefrog brain (Hyla cinerea). Brain Behav Evol 74: 143-154.

-Aste N, Cozzi B, Stankov B, Panzica G (2001): Sexual differences and effect of photoperiod on melatonin receptor in avian brain. $\mathrm{Mi}$ crosc Res Tech 55:37-47.

Axelrod J (1974): The pineal gland: a neurochemical transducer. Science 184:13411348.

Bentley GE, Ball GF (2000): Photoperiod-dependent and -independent regulation of melatonin receptors in the forebrain of songbirds. J Neuroendocrinol 12:745-752.

Boyd SK (1994): Arginine vasotocin facilitation of advertisement calling and call phonotaxis in bullfrogs. Horm Behav 28:232-240.

-Burmeister S, Somes C, Wilczynski W (2001): Behavioral and hormonal effects of exogenous vasotocin and corticosterone in the green treefrog. Gen Comp Endocrinol 122: 189-197.

Burmeister SS, Wilczynski W (2001): Social context influences androgenic effects on calling in the green treefrog (Hyla cinerea). Horm Behav 40:550-558.

Cassone VM, Natesan AK (1997): Time and time again: the phylogeny of melatonin as a transducer of biological time. J Biol Rhythms 12: 489-497.

Chu J, Marler CA, Wilczynski W (1998): The effects of arginine vasotocin on the calling behavior of male cricket frogs in changing social contexts. Horm Behav 34:248-261.

-Clemens JW, Jarzynka MJ, Witt-Enderby PA (2001): Down-regulation of mt1 melatonin receptors in rat ovary following estrogen exposure. Life Sci 69:27-35.

de Vlaming VL, Sage M, Charlton CB (1974): The effects of melatonin treatment on gonadosomatic index in the teleost Fundulus similis, and the tree frog, (Hyla cinerea). Gen Comp Endocrinol 22: 433-438.
Krohmer RW, Lutterschmidt DI (2011): Environmental and neuroendocrine control of reproduction in snakes; in Jamieson BGM (ed): Reproductive Biology and Phylogeny. Boca Raton, CRC Press, vol 9, pp 289-346. nisms, similar endpoints. Neuroscience 138: 947-955.

Godwin J (2010): Neuroendocrinology of sexual plasticity in teleost fishes. Front Neuroendocrinol 31:203-216.

Goodson JL, Bass AH (2001): Social behavior functions and related anatomical characteristics of vasotocin/vasopressin systems in vertebrates. Brain Res Brain Res Rev 35:246265.

-Hillsman KD, Sanderson NS, Crews D (2007): Testosterone stimulates mounting behavior and arginine vasotocin expression in the brain of both sexual and unisexual whiptail lizards. Sex Dev 1:77-84.

Isobe Y, Torii T, Nishino H (2001): Melatonin inhibits Arg-vasopressin release via MT(2) receptor in the suprachiasmatic nucleus-slice culture of rats. Brain Res 889:214-219.

Juszczak M (2001): The hypothalamo-neurohypophysial response to melatonin. Neuro Endocrinol Lett 22:169-174.

Kabelik D, Weiss SL, Moore MC (2008): Arginine vasotocin (AVT) immunoreactivity relates to testosterone but not territorial aggression in the tree lizard, Urosaurus orna tus. Brain Behav Evol 72:283-294.

Lutterschmidt DI (2006): Chronobiology of Garter Snakes: Environmental and Hormonal Cues Mediating Hibernation and Reproduction; doctoral dissertation, Oregon State University, Corvallis.

Lynch KS, Wilczynski W (2005): Gonadal steroids vary with reproductive stage in a tropically breeding female anuran. Gen Comp Endocrinol 143:51-56.

- Marler CA, Chu J, Wilczynski W (1995): Arginine vasotocin injection increases probability of calling in cricket frogs, but causes call changes characteristic of less aggressive males. Horm Behav 29:554-570.

Marler CA, Boyd SK, Wilczynski W (1999): Forebrain arginine vasotocin correlates of alternative mating strategies in cricket frogs. Horm Behav 36:53-61.

Maruska KP, Mizobe MH, Tricas TC (2007): Sex and seasonal co-variation of arginine vasotocin (AVT) and gonadotropin-releasing hormone $(\mathrm{GnRH})$ neurons in the brain of the halfspotted goby. Comp Biochem Physiol A Mol Integr Physiol 147:129-144.

Kalsbeek A, Fliers E, Hofman MA, Swaab DF, Buijs RM (2010): Vasopressin and the output of the hypothalamic biological clock. J Neuroendocrinol 22:362-372.

Kime NM, Whitney TK, Davis ES, Marler CA (2007): Arginine vasotocin promotes calling behavior and call changes in male túngara frogs. Brain Behav Evol 69:254-265.

Kime NM, Whitney TK, Ryan MJ, Rand AS, Marler CA (2010): Treatment with arginine vasotocin alters mating calls and decreases call attractiveness in male túngara frogs. Gen Comp Endocrinol 165:221-228.

Klomberg KF, Marler CA (2000): The neuropeptide arginine vasotocin alters male call characteristics involved in social interactions in the grey treefrog, Hyla versicolor. Anim Behav 59:807-812.
- Mayer I, Bornestaf C, Borg B (1997): Melatonin in non-mammalian vertebrates: physiological role in reproduction? Comp Biochem Physiol A Mol Integr Physiol 118:515-531.

Mendonça MT, Tousignant AJ, Crews D (1996): Pinealectomy, melatonin, and courtship behavior in male red-sided garter snakes (Thamnophis sirtalis parietalis). J Exp Zool 274:63-74.

Mogulkoc R, Baltaci AK (2010): Effect of melatonin supplementation on plasma vasopressin response to different conditions in rats with hyperthyroidism induced by L-thyroxine. Regul Pept 161:38-42.

Neary TJ, Northcutt RG (1983): Nuclear organization of the bullfrog diencephalon. J Comp Neurol 213:262-278. 
Northcutt RG, Kicliter E (1980): Organization of the amphibian telencephalon; in Ebbesson SOE (ed): Comparative Neurology of the Telencephalon. New York, Plenum Press, pp 203-255.

O’Bryant EL, Wilczynski W (2010): Changes in plasma testosterone levels and brain AVT cell number during the breeding season in the green treefrog. Brain Behav Evol 75:271281.

Penna M, Capranica RR, Somers J (1992): Hormone-induced vocal behavior and midbrain auditory sensitivity in the green treefrog, Hyla cinerea. J Comp Physiol A 170:73-82.

- Plumari L, Viglietti-Panzica C, Allieri F, Honda S, Harada N, Absil P, Balthazart J, Panzica GC (2002): Changes in the arginine-vasopressin immunoreactive systems in male mice lacking a functional aromatase gene. J Neuroendocrinol 14:971-978.

Prendergast BJ (2005): Internalization of seasonal time. Horm Behav 48:503-511.

Propper CR, Dixon TB (1997): Differential effects of arginine vasotocin and gonadotropin-releasing hormone on sexual behaviors in an anuran amphibian. Horm Behav 32: 99-104.
Rasri K, Mason P, Govitrapong P, Pevet P, Klosen $P$ (2008): Testosterone-driven seasonal regulation of vasopressin and galanin in the bed nucleus of the stria terminalis of the Djungarian hamster (Phodopus sungorus). Neuroscience 157:174-187.

Revel FG, Masson-Pevet M, Pevet P, Mikkelsen JD, Simonneaux V (2009): Melatonin controls seasonal breeding by a network of hypothalamic targets. Neuroendocrinology 90 : $1-14$.

Semsar K, Klomberg KF, Marler C (1998): Arginine vasotocin increases calling-site acquisition by nonresident male grey treefrogs. Anim Behav 56:983-987.

-Serino I, Izzo G, Ferrara D, Minucci S, d'Istria M (2011): First evidence of a cDNA encoding for a melatonin receptor (mel 1b) in brain, retina, and testis of Pelophylax esculentus. J Exp Zool A Ecol Genet Physiol 315:520-526.

-Skene DJ, Masson-Pévet M, Pévet P (1993): Seasonal changes in melatonin binding sites in the pars tuberalis of male European hamsters and the effect of testosterone manipulation. Endocrinology 132:1682-1686.
Tamarkin L, Baird CJ, Almeida OF (1985): Melatonin: a coordinating signal for mammalian reproduction? Science 227:714-720.

Tavolaro R, Canonaco M, Franzoni MF (1995): Comparison of melatonin-binding sites in the brain of two amphibians: an autoradiographic study. Cell Tissue Res 279:613-617.

Tito MB, Hoover MA, Mingo AM, Boyd SK (1999): Vasotocin maintains multiple call types in the gray treefrog, Hyla versicolor. Horm Behav 36:166-175.

-Wiechmann AF, Campbell LD, Defoe DM (1999): Melatonin receptor RNA expression in Xenopus retina. Mol Brain Res 63:297303.

Wiechmann AF, Hollaway LR, Rada JA (2009): Melatonin receptor expression in Xenopus laevis surface corneal epithelium: diurnal rhythm of lateral membrane localization. Mol Vis 15:2384-2403.

Wiechmann AF, Vrieze MJ, Wirsig-Wiechmann CR (2003): Differential distribution of melatonin receptors in the pituitary gland of Xenopus laevis. Anat Embryol (Berl) 206: 291-299.

-Wilczynski W, Lynch KS, O’Bryant EL (2005): Current research in amphibians: studies integrating endocrinology, behavior, and neurobiology. Horm Behav 48:440-450. 